\title{
Explosive events in the solar atmosphere seen in extreme-ultraviolet emission lines
}

\author{
J. E. Mendoza-Torres ${ }^{1}$, J. P. Torres-Papaqui ${ }^{1}$, and K. Wilhelm ${ }^{2}$ \\ ${ }^{1}$ Instituto Nacional de Astrofisica, Optica y Electrónica, Puebla, Mexico \\ e-mail: mend@inaoep.mx \\ 2 Max-Planck-Institut für Aeronomie, 37191 Katlenburg-Lindau, Germany
}

Received 15 May 2004 / Accepted 5 October 2004

\begin{abstract}
We present observations of explosive events (EEs) in the solar atmosphere obtained with the Solar Ultraviolet Measurements of Emitted Radiation (SUMER) spectrometer on the Solar and Heliospheric Observatory (SOHO) in the wavelength range from about $750 \AA$ to $790 \AA$. Prominent spectral lines in this range are emitted by ions which have temperatures of maximum ionic abundances between $1.0 \times 10^{5} \mathrm{~K}$ and $6.3 \times 10^{5} \mathrm{~K}$ in ionization equilibrium, and are therefore expected to be formed in the transition region (TR) and in the low corona. The aim of this work is to investigate whether the EEs originate in a limited range of temperatures or in a wide interval. We analyzed the behaviour of several emission lines during 114 EEs. In many events, the radiance increased first in lines with formation temperatures near $1.5 \times 10^{5} \mathrm{~K}$. A number of events produced profiles that clearly revealed blue and red components, in addition to the central line. In general, both the radiance and the lineof-sight (LOS) velocity of the blue component are larger than those of the red one. From an inspection of the profiles that did not show all three spectral components, we found, in all the cases, that the lowest temperature line showed a red shift whereas the highest temperature was characterized by a blue shift. The inverse situation was not observed. We interpret these results as an indication that most of the EEs originate at intermediate temperatures of the TR as fast reconnection jets.
\end{abstract}

Key words. Sun: transition region - Sun: activity - Sun: UV radiation - Sun: atmosphere

\section{Introduction}

The processes governing the energy input into the corona and its subsequent heating still pose unresolved questions. Based on observations of the density-sensitive O IV $\lambda \lambda 1401,1399$ line ratio, Judge et al. (1998) found evidence for downwards travelling waves and suggested that this could be a signature of coronal heating by nanoflare activity consistent with Parker's (1988) picture of discrete heating events. Explosive events (EEs), also of a discrete nature, have been observed in a very wide range of temperatures, from about $2 \times 10^{4} \mathrm{~K}$ to one million Kelvin (Brueckner \& Bartoe 1983) corresponding to the transition region (TR) and the low corona. EEs are mainly characterized by red and blue Doppler-shifted components of the emission line profile. Some observational results suggest that EEs have a chromospheric origin (Teriaca et al. 2002), and Dere (1994) found that EEs seem to be located just above the photosphere. On the other hand, no observable coronal counterpart to EE has been detected (Moses \& Cook 1994).

In quiet-Sun regions, an average red shift has been observed in most of the TR lines (Doschek et al. 1976; Brekke et al. 1997; Chae et al. 1998a), whereas coronal lines exhibit on average blue shifts, according to recent studies (Peter \& Judge 1999; Dammasch et al. 1999). A model assuming the occurrence of nanoflares at a temperature of $3 \times 10^{5} \mathrm{~K}$ reproduced the observed shifts quite well (Teriaca et al. 1999). Models that assume an energy source below the TR also reproduced well the blue and red shifts (Karpen et al. 1995; Sarro et al. 1999) and the bright and wide central component observed at the first stages (Sarro et al. 1999).

The temperature at which an EE originates (and consequently the approximate altitude) plays an important role in determining the amount of energy transported between different layers of the solar atmosphere (Innes \& Tóth 1999). Therefore, not only the fact that EEs are observed in the TR, but also the temperature at their origin is significant in estimating their importance for heating the corona. However, up to now it is still not clear whether or not the EEs originate at a given temperature, and also how far away their influence may reach from that initial temperature regime.

The analysis of EEs in spectral lines formed at different temperatures may provide some insight into the question whether EEs are generated more frequently at a given temperature or not. The behaviour of the EEs at different TR temperatures may also help in estimating the possible input of energy from EEs to solar atmospheric heating. In this work, we will therefore study the onset of EEs as a function of the formation temperature of the emission lines in which they were observed. 
Table 1. Wavelengths, electron temperatures of maximum ionic fractions and formation temperatures of the lines analyzed with our selection routine.

\begin{tabular}{lccc}
\hline \hline Line & Wavelength $/ \AA$ & $T_{\max } / \mathrm{K}$ & $T_{\text {form }} / \mathrm{K}$ \\
\hline S IV & 750.2 & $1.0 \times 10^{5}$ & $1.1 \times 10^{5}$ \\
N IV & 765.1 & $1.4 \times 10^{5}$ & $1.5 \times 10^{5}$ \\
S V & 786.4 & $1.5 \times 10^{5}$ & $1.6 \times 10^{5}$ \\
O IV & 787.7 & $1.5 \times 10^{5}$ & $1.6 \times 10^{5}$ \\
O V & 760.4 & $2.3 \times 10^{5}$ & $2.3 \times 10^{5}$ \\
Ne VIII & 770.4 & $6.3 \times 10^{5}$ & $6.2 \times 10^{5}$ \\
Ne VIII & 780.3 & $6.3 \times 10^{5}$ & $6.2 \times 10^{5}$ \\
\hline
\end{tabular}

\section{Observations and data analysis}

The data were acquired with the Solar Ultraviolet Measurements of Emitted Radiation (SUMER) spectrometer on the Solar and Heliospheric Observatory (SOHO) (Wilhelm et al. 1997) from 14 to 16 November 1996. The time intervals covered by the observations are $4 \mathrm{~h}, 2 \mathrm{~h}$ and $8 \mathrm{~h}$, respectively for 14,15 and 16 November giving a total of $14 \mathrm{~h}$ of observations. Seen from SOHO, the angular solar radius was 980" (one second of an arc corresponds to $710 \mathrm{~km}$ ). The observations were performed in the wavelength range from about $750 \AA$ to $790 \AA$, which contains emission lines with widely different formation temperatures. The spectral resolution element was $0.045 \AA$ in the first order.

The spectrometer slit was directed towards quiet-Sun areas on the solar disk in different observing sequences. In all the sequences the slit was oriented along the central meridian, but at different N-S locations. On 14 November, in the first set of observations, the center of the slit was directed to $810^{\prime \prime}$ south and in the next one to $560^{\prime \prime}$ south. On 15 November, it was pointed to $810^{\prime \prime}$ north and to $560^{\prime \prime}$ north one day later.

The slit was not adjusted to follow the solar rotation during these sequences. A region was first observed with a slit of $4 "$ width and $300^{\prime \prime}$ length in a $30 \mathrm{~s}$ cadence for about $5 \mathrm{~min}$ until the SUMER mass memory was full. After that time the same region, only slightly moved by the solar rotation, was observed with the $1^{\prime \prime} \times 300^{\prime \prime}$ slit in a 570 s cadence. The second phase was necessary to clear the memory of the data accumulated in the first phase.

The time evolution of EEs was either partially or fully recorded. From these observations we selected those EEs that were observed from the beginning in the $30 \mathrm{~s}$ cadence data in the lines listed in Table 1 which were the lines that showed the most significant enhancements.

In order to analyze this large amount of data we made the first selection of possible EEs with a computer routine. Before running this routine we selected the most intense lines identified from the spectra integrated over each day. Based on this selection, we could analyze the lines in Table 1, listed together with the wavelength, the electron temperature of maximum ionic abundance taken from Mazzotta et al. (1998), and the corresponding formation temperatures (Mason \& Monsignori Fossi 1994) of each line.

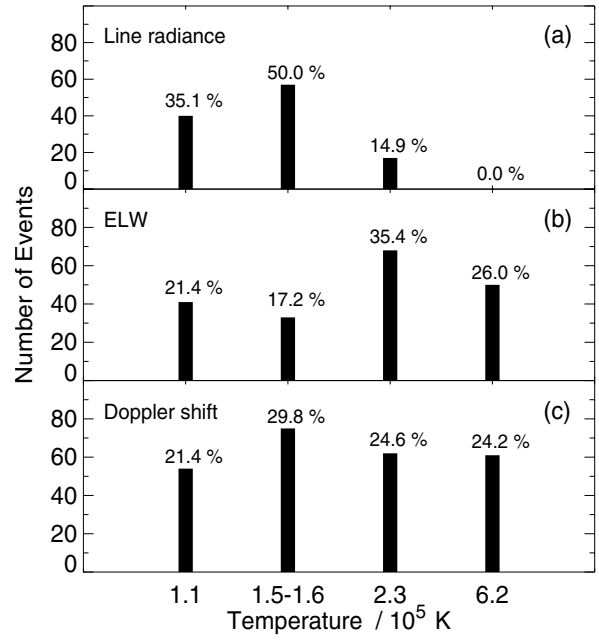

Fig. 1. a) Histogram of the number of events that reached first the $3 \sigma$ level threshold above quiescence in a given spectral line. Also given are the corresponding relative occurrence frequencies. b) The corresponding histograms for the ELW; and c) for the Doppler shifts. The three lines formed near $1.5 \times 10^{5} \mathrm{~K}$ are binned and shown only once, so are the Ne VIII lines at $6.2 \times 10^{5} \mathrm{~K}$.

The method used to identify EEs was as follows: (1) based on the spectra integrated over each day we identified the darkest spatial regions at each spectral line in Table 1 . The extension of these regions was typically of 3-4 spatial pixels. (2) We then computed the mean line spectral radiance and the standard deviation $(1 \sigma)$ of the amplitude of the line at these spatial segments. For the amplitude we used the maximum of the line profile and the amplitudes of the two neighbouring points, one point at each side. (3) One would expect that without EE the variations would be smaller than $3 \sigma$ at any time. Therefore, the routine was designed to determine the times when the radiance of each line attained the $3 \sigma$ value allowing us to identify, for each of the events, which line reached this value first. In most of cases, there is one temporal resolution element lag (that is $30 \mathrm{~s}$ ) between the $3 \sigma$ detections in the first and subsequent lines. The results for the 30 s cadence are shown in Fig. 1a. (4) The line radiances of each line at some spatial pixels - where an EE was later observed - were measured repeatedly for some time interval prior to an event. We then compared the line shape for times prior to and during the events, and confirmed by inspection of the line profiles that an EE had been detected. (5) For each line profile we also estimated its width. For a spectral line one can roughly estimate the line width using the area under the line $(S)$ and the maximum excess of the line $(A)$. The Equivalent Line Width (ELW) is the width of the rectangle of height $A$ and surface $S$. Therefore the ELW is simply the ratio between $S$ and $A$. For each profile we measured the maximum amplitude. Based on the observed profile, we computed the area under the line and then the ELW. With a procedure similar to that employed for the line radiance, we determined, for each event, the lines that first attained an ELW increase of $3 \sigma$ (Fig. 1b). (6) The wavelength of the maximum of a line was considered to establish the central wavelength. Finally, we determined the pre-EE standard deviation of the Doppler shift and the corresponding EE-related wavelength shifts in either spectral direction. 
Table 2. Line-of-sight (LOS) velocities of the jets in various emission lines and the electron densities (from O v line ratios) observed in November 1996 together with an indication in the last column whether the $3 \sigma$ radiance level was reached first in this specific line. The asterisk denotes an EE that showed all three components in both lines listed. The times given correspond to the time when the radiance attained the $3 \sigma$ value.

\begin{tabular}{|c|c|c|c|c|c|c|c|}
\hline \multirow[t]{2}{*}{ Line } & \multirow{2}{*}{$\begin{array}{l}\lambda / \\
\AA \\
\AA\end{array}$} & \multirow{2}{*}{$\begin{array}{l}\text { Time / UTC } \\
\text { HH:MM:SS }\end{array}$} & \multirow{2}{*}{$\begin{array}{c}v_{\text {blue }} / \\
\mathrm{km} \mathrm{s}^{-1}\end{array}$} & \multirow{2}{*}{$\begin{array}{c}v_{\text {red }} / \\
\mathrm{km} \mathrm{s}^{-1}\end{array}$} & \multicolumn{2}{|c|}{$n_{\mathrm{e}} / 10^{9} \mathrm{~cm}^{-3}$} & \multirow[t]{2}{*}{ Category } \\
\hline & & & & & core & red & \\
\hline \multicolumn{8}{|c|}{ November 14} \\
\hline S IV & 750.2 & $12: 14: 18$ & -52 & 50 & 12 & - & yes \\
\hline $\mathrm{Ne}$ VIII & 770.4 & $12: 15: 18$ & -57 & 50 & 10 & - & no \\
\hline O IV & 787.7 & $12: 18: 48$ & -54 & 50 & 54 & 500 & yes \\
\hline S IV & 750.2 & $12: 19: 48$ & -63 & 50 & 8.6 & - & yes \\
\hline O IV* & 787.7 & $14: 41: 55$ & -52 & 50 & 31 & - & yes \\
\hline $\mathrm{Ne} \mathrm{VIII}^{*}$ & 770.4 & $14: 41: 55$ & -61 & 46 & 2.4 & 91 & no \\
\hline $\mathrm{O} \mathrm{V}$ & 760.4 & $14: 42: 25$ & -73 & 72 & 2.8 & 54 & yes \\
\hline \multicolumn{8}{|c|}{ November 15} \\
\hline $\mathrm{Ne}$ VIII & 770.4 & $12: 05: 57$ & -58 & 51 & 6.4 & 190 & no \\
\hline O IV & 787.7 & $12: 06: 57$ & -55 & 53 & 30 & - & yes \\
\hline S IV & 750.2 & 12:07:57 & -62 & 52 & 2.0 & - & yes \\
\hline O IV & 787.7 & $12: 08: 27$ & -56 & 52 & 6.3 & - & yes \\
\hline \multicolumn{8}{|c|}{ November 16} \\
\hline $\mathrm{OV}$ & 760.4 & 6:37:56 & -55 & 53 & 29 & - & yes \\
\hline S IV & 750.2 & 6:48:41 & -52 & 50 & 31 & - & yes \\
\hline O IV & 787.7 & 6:49:41 & -53 & 48 & 32 & 220 & yes \\
\hline O IV & 787.7 & 6:50:41 & -55 & 48 & 28 & 360 & yes \\
\hline O IV & 787.7 & 6:51:11 & -49 & 49 & 3.9 & 260 & yes \\
\hline $\mathrm{OV}$ & 760.4 & $7: 23: 28$ & -51 & 50 & 2.4 & - & yes \\
\hline $\mathrm{OV}$ & 760.4 & 9:15:13 & -60 & 48 & 22 & - & yes \\
\hline $\mathrm{Ne}$ VIII & 770.4 & $9: 17: 43$ & -58 & 48 & 2.3 & - & no \\
\hline $\mathrm{Ne}$ VIII & 770.4 & 9:18:13 & -52 & 52 & 6.3 & 220 & no \\
\hline S IV & 750.2 & $9: 31: 00$ & -52 & 50 & 32 & - & yes \\
\hline $\mathrm{Ne}$ VIII & 770.4 & $11: 43: 45$ & -53 & 47 & 25 & 280 & no \\
\hline $\mathrm{Ne}$ VIII & 770.4 & 11:44:15 & -50 & 50 & 2.6 & - & no \\
\hline
\end{tabular}

The number of events for which a certain line first reached Doppler shifts of $3 \sigma$ is shown in Fig. 1c. We did not distinguish between the N IV $\lambda 765.1, \mathrm{~S}$ V $\lambda 786.4$, and O IV $\lambda 787.7$ lines with very similar formation temperatures, but computed an average value as follows: the number of events where at least one of these lines reached the $3 \sigma$ level first were added and divided by three. Only this average is plotted in Fig. 1. Similarly, both Ne VIII sets were combined. We defer further discussion of Fig. 1 to Sect. 3.

We also looked at the $570 \mathrm{~s}$ observations. EEs are, however, too short-lived to obtain results other than temporallyintegrated spectra from this long integration time.

Prior to an EE, the line profiles had nearly Gaussian shapes and they were deformed as the radiance grew and Doppler shifts were detected. Some of these events were observed only in one line. On the other hand, we considered that an EE was detected in two or more lines when the time interval of the growing phase of an event, in a given line, overlapped with the same phase of an event in another line. Taking into account this criterion, a total of 114 events were identified. For 22 of them, the line shape of at least one spectral line observed prior to an EE could be separated into three Gaussian curves. These events are compiled in Table 2. Only in one of these 22 cases the three spectral components were observed at two lines. This means that 23 line profiles could be separated into three spectral components. The deblending was made with an IDL routine using three Gaussians. In the other 92 EEs the line shape was neither a Gaussian curve alone nor the sum of three Gaussians. By measuring the wavelength of the maximum of the line, we found that in all of these cases a Doppler shift was evident.

In order to estimate the electron density during an EE, we performed computations using the O $\mathrm{V} \lambda 759.4 / \lambda 761.2$ line radiance ratio (Curdt et al. 1997). For both lines, we tried to measure the radiance (in photon units) of the three spectral components: the core line as well as the red and blue contributions in analogy to Fig. 2. The O v $\lambda 759.4$ line did not pose any difficulties. In all events the central component of the O $\mathrm{V} \lambda 761.2$ line was also bright enough to determine its radiance. Gaussian fits allowed us to determine the radiance of the red components of this line in nine cases. The blue wing was just above the noise level and could not be separated. The electron densities so obtained are also given in Table 2. We checked whether the events actually reached the $3 \sigma$ radiance levels first in the lines listed (the cases where the $3 \sigma$ level of the radiance was attained first 

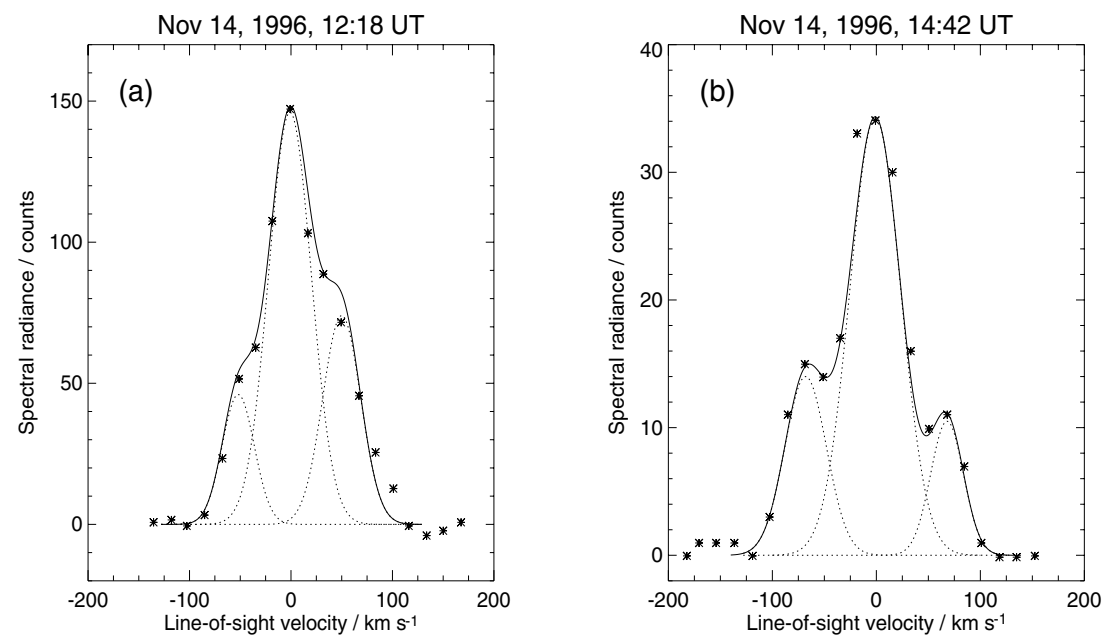

Fig. 2. Two different profiles a) the O IV $\lambda 787.7$ line b) the O v $\lambda 760.4$ line after separation into Gaussians. Asterisks denote the observational data while the dotted lines the fitted Gaussians and the continuous line the sum of the Gaussians. The profiles do not belong to the same event, but to rows 3 and 7 of Table 2 .

Table 3. Average radiances and velocities for the 23 line profiles that could be separated into three components.

\begin{tabular}{lcccccccr}
\hline \hline & $R(1.1)$ & $\operatorname{std}(1.1)$ & $R(1.6)$ & $\operatorname{std}(1.6)$ & $R(2.3)$ & $\operatorname{std}(2.3)$ & $R(6.2)$ & $\operatorname{std}(6.2)$ \\
\hline Blue & 7.1 & 3.9 & 19.7 & 13.2 & 22.5 & 13.9 & 12.8 & 5.0 \\
Central & 16.6 & 8.1 & 51.2 & 32.0 & 54.0 & 36.2 & 33.4 & 12.1 \\
Red & 3.9 & 1.7 & 14.4 & 8.3 & 13.5 & 11.1 & 9.5 & 3.8 \\
\hline & $V(1.1)$ & $\operatorname{std}(1.1)$ & $V(1.6)$ & $\operatorname{std}(1.6)$ & $V(2.3)$ & $\operatorname{std}(2.3)$ & $V(6.2)$ & $\operatorname{std}(6.2)$ \\
\hline Blue & 55.9 & 6.0 & 53.6 & 2.3 & 62.8 & 7.2 & 53.9 & 3.2 \\
Red & 50.5 & 1.2 & 49.9 & 1.8 & 53.9 & 12.3 & 50.2 & 2.1 \\
\hline
\end{tabular}

at the given line are indicated with "yes"). This was the case for all lines except Ne VIII. To get some idea of the uncertainties involved in the computation of the density, we performed several Gaussian fits of the same profile with different input conditions concerning the background estimate. The fits for which the width was either very wide or very narrow were excluded. The ratios obtained for the remaining fits were averaged. The resulting values were transformed into density. The differences between averaged and individual values were less than $20 \%$.

\section{Results}

The most significant result from Fig. 1 is that for none of the events the radiance enhancements at the highest temperature preceded the enhancement of other lines, and that, in about $50 \%$ of the cases, lines with formation temperatures near $1.5 \times 10^{5} \mathrm{~K}$ increased first (Fig. 1a). On the other hand, most of the ELW increases and many of the Doppler shifts first happened at higher temperatures (Figs. 1b and 1c). The increase in ELW was observed one temporal resolution element later than the radiance increase.

The profiles that could be separated into three Gaussians were observed near the beginning of the EEs. In these cases, we identified the spectral components by Gaussian fits (Fig. 2). After that we determined the radiance, the LOS velocity and the line width for each of them. The deduced LOS velocities of the blue and red spectral components are given in Table 2. The averages of the radiances and the averages of the velocities (over all the 23 line profiles) are given in Table 3 together with the standard deviations. In parenthesis, the formation temperature of the line in units of $10^{6} \mathrm{~K}$ is indicated. The radiances in Table 3 are given in units of $10^{17}$ photons s${ }^{-1} \mathrm{~cm}^{-2} \mathrm{sr}^{-1}$. In none of the events did the N IV and S V lines exhibit profiles such as those in Figs. 2a and 2b, and, therefore, no spectral components could be determined for these lines. As may be seen from the examples in Fig. 2, the LOS velocity of the blue components have larger values than those of the red ones. This is a general trend (Table 3). Specifically for the Ne VIII line, the velocity of the blue component is larger than the red one for all events. The velocity of the central component was considered to be zero. Table 3 shows that the radiance of the blue component also seems to have a tendency to be larger than that of the red component. However, this is not always the case (as may be seen from Fig. 2a).

As mentioned above, in 92 cases the line profiles did not have resolvable satellite components, but showed radiance increases, line broadenings and departures from the shape of a Gaussian curve. In all these cases the lowest-temperature line $\left(1.1 \times 10^{5} \mathrm{~K}\right)$ was red shifted whereas the highest-temperature line $\left(6.2 \times 10^{5} \mathrm{~K}\right)$ was blue shifted (Fig. 3). In most of the cases the shifts ocurred at $40-70 \mathrm{~km} \mathrm{~s}^{-1}$ for the Ne VIII line and $50-80 \mathrm{~km} \mathrm{~s}^{-1}$ for the S IV one. The opposite situation was not observed.

The electron density for the core spectral component averaged over the 22 events is $n_{\mathrm{e}}=1.7 \times 10^{10} \mathrm{~cm}^{-3}$. Estimates of the electron density of the quiet Sun by Pérez et al. (1999) 


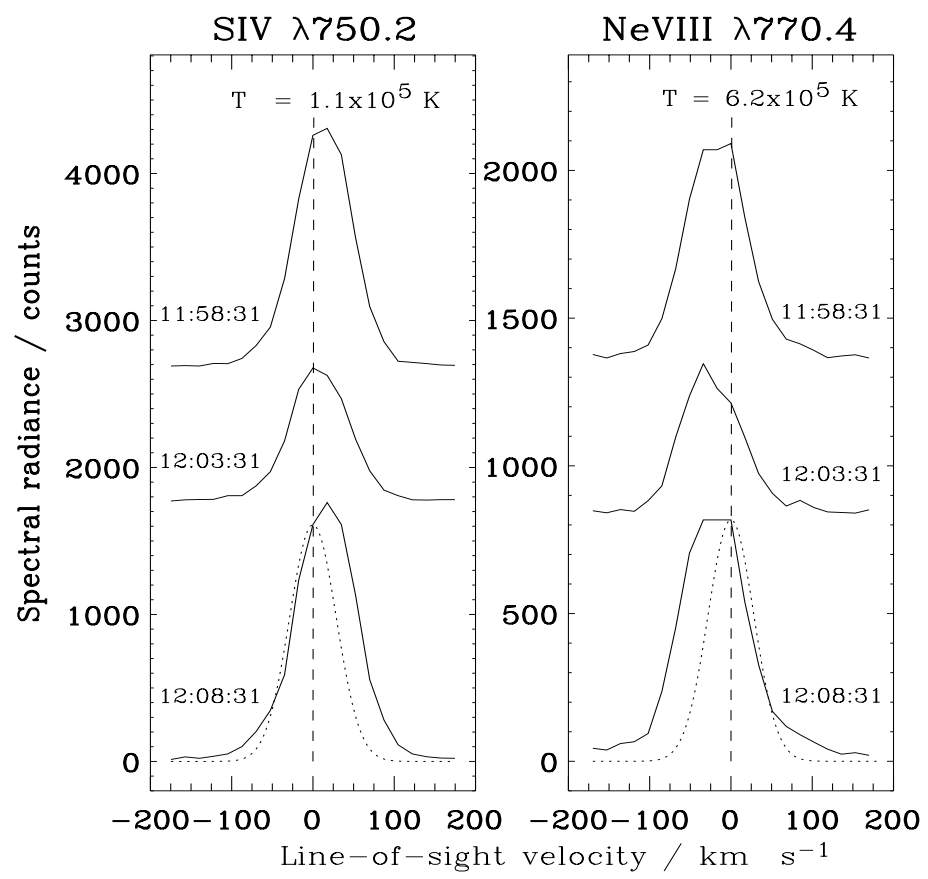

Fig. 3. Profiles of the lines of extreme temperatures observed during an EE. It may be seen that the S IV $\lambda 750.2$ line is shifted to the red while the Ne VIII $\lambda 770.4$ line is shifted to the blue. Dotted lines denote the line profiles before the EEs (amplified 27 and 17 times, respectively) for comparison. The upper profiles have been offset for clarity.

led to values between $3.6 \times 10^{9} \mathrm{~cm}^{-3}$ and $1.2 \times 10^{11} \mathrm{~cm}^{-3}$. Landi et al. (2000) based on the O v $\lambda 629.8 / \mathrm{NV} \lambda 1238.8$ ratio also found densities of the order of $10^{10} \mathrm{~cm}^{-3}$. Estimates of the electron density for EEs gave values of the same order (Brueckner \& Bartoe 1983). Therefore, the estimated average density of the core component supports values published in the literature, whereas the red components have much higher densities suggesting the lower altitudes of their formation.

Our density determinations based on a single species, namely $\mathrm{O}^{4+}$ ions, give values that are relatively independent of ionization equilibrium assumptions, but may nevertheless be biased during such dynamic events. However, the large change from the core to the red component provides some confidence that the latter is indeed emitted from denser regions, and with the downward motion indicated by the red Doppler shift, from lower altitudes.

\section{Discussion}

In nearly half of the cases studied here, the radiance increases related to EEs first occurred for spectral lines with formation temperatures of about $1.5 \times 10^{5} \mathrm{~K}$. None of the events showed such an initial increase at $6.2 \times 10^{5} \mathrm{~K}$. A firm conclusion that can be drawn from these findings is that our EEs are not caused by radiative cooling of thermally instable coronal plasma suggested as the cause of certain transient events observed in C IV at $1 \times 10^{5} \mathrm{~K}$ (Dere et al. 1981). However, they are consistent with an initial energy release in cooler plasmas that are heated very fast to transition-region temperatures. Many studies have demonstrated that this energy release is in all likelihood related to magnetic reconnection. In particular, Dere et al. (1991) have shown that EEs occur during photospheric magnetic flux cancellations, a relationship studied in detail by Chae et al. (1998b) using SUMER Si IV observations and Big Bear Solar Observatory magnetograms. Blue and red jets originating from EE locations seen by Innes et al. (1997) support the idea of a reconnection process, the details of which are nevertheless not yet known.

Magnetic reconnection thus appears to be the best working hypotheses for the generation of EEs, which we will use for our discussion. We have seen that the radiative effects of the process start at transition-region temperatures near $1.5 \times 10^{5} \mathrm{~K}$, but Doppler velocities can, in most cases, first be detected at higher temperatures. This is in line with the notion that we do not see the initial fast reconnection jet, which should have higher velocities (close to the Alfvén speed), but rather, secondary effects in the shocked plasma.

In Table 2 there is no event with red and blue spectral components in the N IV or S V lines with formation temperatures near $1.5 \times 10^{5} \mathrm{~K}$. Although our statistics are rather poor, this might indicate that the plasma is heated before we can detect Doppler velocities. The O IV line that shows the shifted components has a much wider contribution function than the N IV and S V lines. More surprising is the observation of red and blue components in the Ne VIII line. This might suggest that the blue jet is not just extending to high temperatures, but that the bi-directional acceleration process is affecting plasmas of higher temperatures in the course of an event. More theoretical work is required to substantiate this suggestion. In this context it should be recalled that the plasma temperature is only an approximate indication of the altitude; in fact, it has been shown that the height range of transition-region (N IV, O IV, O V) and Ne VIII emissions overlap to a large extent in the solar atmosphere (Wilhelm et al. 2002). 
In a relative sense, we can however derive from the lineratio measurement in $\mathrm{OV}$ that the red component originates from denser (and thus lower) regions than the core of the line. Unfortunately, we do not have the corresponding information on the blue component available, but even without this confirmation it can be concluded that the origins of the core line and one satellite line are not at the same altitude. Using our concept of the fast reconnection jets described above, we could say that the red (downward) jet is accelerating the plasma at lower altitudes.

Finally, we have to consider those cases in which we do not resolve the spectral components. Many of them display a general blue shift of the lines at high temperatures and a red shift at low temperatures. Although it is difficult to draw specific conclusions from these observations, they are in general agreement with the idea that the energy release takes place at intermediate temperatures.

Acknowledgements. We would like to thank D. Germerott for his help in the data handling and calibration. We also acknowledge partial support from CONACyT (Grant No. 1433PT). SUMER is part of SOHO, the Solar and Heliospheric Observatory of ESA and NASA. We also thank the referee, L. M. Sarro, for his useful comments which improved this paper.

\section{References}

Brekke, P., Hassler, D. M., \& Wilhelm, K. 1997, Sol. Phys., 175, 349 Brueckner, G. E., \& Bartoe, J.-D. F. 1983, ApJ, 272, 329

Chae, J., Yun, H. S., \& Poland, A. I. 1998a, ApJS, 114, 151

Chae, J., Wang, H., Lee, C.-Y., Goode, P.R., \& Schühle, U. 1998b, ApJ, 497, L109
Curdt, W., Feldman, U., Laming, J. M., et al. 1997, A\&AS, 126, 281

Dammasch, I. E., Wilhelm, K., Curdt, W., \& Hassler, D. M. 1999, A\&A, 346, 285

Dere, K. P. 1994, Adv. Space Res., 14, (4) 13

Dere, K. P., Bartoe, J.-D. F., Brueckner, G. E., Dykton, M. D., \& VanHoosier, M. E. 1981, ApJ, 249, 333

Dere, K. P., Bartoe, J.-D. F., Brueckner, G. E., Ewing, J., \& Lund, P. 1991, J. Geophys. R., 96, 9399

Doschek, G. A., Feldman, U., \& Bohlin, J. D. 1976, ApJ, 205, L177

Innes, D. E., Inhester, B., Axford, W. I., \& Wilhelm, K. 1997, Nature, 386,811

Innes, D. E., \& Tóth, G. 1999, Sol. Phys., 185, 127

Judge, P. G., Hansteen, V., Wikstøl, Ø, et al. 1998, ApJ, 502, 981

Karpen, J. T., Antiochos, S. K., \& De Vore, C. R. 1995, ApJ, 450, 422

Landi, E., Mason, H. E., Lemaire, P., \& Landini, M. 2000, A\&A, 357, 756

Mason, H. E., \& Monsignori Fossi, B. C. 1994, A\&AR, 6, 123

Mazzotta, P., Mazzitelli, G., Colafrancesco, S., \& Vittorio, N. 1998, A\&AS, 133, 403

Moses, D., \& Cook, J. W. 1994, Space Sci. Rev., 70, 81

Parker, E. N. 1988, ApJ, 339, 474

Pérez, M. E., Doyle, J. G., O’Shea, E., \& Keenan E. P. 1999, A\&A, 351,1139

Peter, H., \& Judge, P. G. 1999, ApJ, 522, 1148

Sarro, L. M., Erdélyi, R., Doyle, J. G., \& Pérez, M. E. 1999, A\&A, 351,721

Teriaca, L., Doyle, J. G., Erdélyi, R., \& Sarro, L. M. 1999, A\&A, 352, L99

Teriaca, L., Madjarska, M. S., \& Doyle, J. G. 2002, A\&A, 392, 309

Wilhelm, K., Inhester, B., \& Newmark, J. S. 2002, A\&A, 382, 328

Wilhelm, K., Lemaire, P., Curdt, W., et al. 1997, Sol. Phys., 170,75 